\title{
A therapeutic trial of acupuncture in neurogenic bladder of spinal cord injured patients - a preliminary report
}

\author{
Pao-Tsai Cheng ${ }^{1}$, May-Kuen Wong $^{1}$ and Phei-Lang Chang ${ }^{2}$ \\ Departments of ${ }^{1}$ Rehabilitation Medicine and ${ }^{2}$ Urology, Chang Gung Memorial Hospital, Chang Gung University, \\ Taipei, Taiwan, R.O.C.
}

Eighty patients with spinal cord injury (SCI) and neurogenic bladder were studied. Among them, $28(70 \%)$ cases in the control group and $32(80 \%)$ cases in the electroacupuncture group achieved ultimately balanced voiding and were selected for further analysis. The acupuncture group received electroacupuncture at four acupoints: Chung Chi (Conception Vessel CV3), Kuan Yuan (CV4), and bilateral Tzu Liao (Urinary Bladder UB32), in addition to conventional intermittent catheterization program (ICP). Whereas the control group underwent conventional bladder training program with ICP only. The results revealed that the time of achieving balanced voiding was statistically significantly shorter with electroacupuncture than in the control group: $57.1 \pm 22.5$ vs $85.2 \pm 27.4$ days $(P<0.005)$ for upper motor neuron lesions, and $55.4 \pm 22.6$ vs $83.4 \pm 26.1$ days $(P<0.01)$ for lower motor neuron lesions. However, there was almost no difference between upper motor neuron lesions and lower motor neuron lesions. When acupuncture commenced was also a factor to influence the results. Those who received acupuncture within 3 weeks after injury had significantly shortened the total days to achieve a balanced bladder, as compared to those who received acupuncture 3 weeks after injury $(46.6 \pm 13.2$ vs $65.8 \pm 15.4$ days, $P<0.005)$. Our study implied that acupuncture might be beneficial in the management of neurogenic bladder of SCI, and the earlier the patient received electro-acupuncture therapy, the sooner the bladder balanced. On the other hand, we also found that complete spinal cord injury, either with pronounced detrusor-sphincter dyssynergia in upper motor nueron lesion or with persistent areflexic bladder in lower motor neuron lesion, was not affected by acupuncture.

Keywords: spinal cord injury; neurogenic bladder; electro-acupuncture; intermittent catheterization program; balanced bladder

\section{Introduction}

Neurogenic bladder is a major problem for spinal cord injured patients. A successful bladder training may not only decrease the mobility or mortality of these patients, but also improve the quality of life and psychological outlook. However, it usually takes two to three months of bladder training to reach balanced status, and only about two thirds can reach balanced bladder by conventional bladder program. ${ }^{1,2}$ Although clean intermittent catheterization was considered as a safe technique for long term management of voiding disorders, owing to the high humidity climate and running water system here in Taiwan, most of our patients would prefer to have catheter-free voiding as soon as possible, especially at home.

In order to achieve control of micturition, different modalities of electrical stimulation have been devised including transurethral intravesical, ${ }^{3}$ transrectal, ${ }^{4}$ transvaginal, ${ }^{5}$ spinal sacral roots, ${ }^{6-8}$ conus medul-

Correspondence: P-T Cheng laris, ${ }^{9}$ and direct pudendal nerve electrical stimulation. ${ }^{10}$ No conclusive results were obtained. Acupuncture, a traditional Chinese therapeutic technique, has been used to treat a great variety of diseases and symptoms-including micturition problems-for more than 2000 years. In this study, we tried to use electroacupuncture for the management of neurogenic bladder in spinal cord injured patients.

\section{Materials and methods}

Eighty spinal cord injured patients with neurogenic bladder hospitalized in the spinal unit and rehabilitation ward of Chang Gung Memorial Hospital were included in this study. According to ASIA (American Spinal Injury Association) of Frankel classification, 45 were Frankel A SCI patients and 35 were Frankel B patients. They all had no motor function below their injury level. No head injury or pelvic fracture was accompanied with them. They were randomly divided into two groups - the control and acupuncture group. Patients in the control group received conventional 
bladder training program including intermittent catheterization, tapping the suprapubic area and trigger point stimulation. Medication such as Diazepam or Phenoxybenzamine was used for relaxation of the urethral sphincter in cases of non-relaxing sphincter or detrusor-sphincter dyssynergia. Whereas in the acupuncture group, electroacupuncture was given in addition to conventional bladder training program. Acupuncture is a traditional Chinese therapeutic technique, most of our patients are quite familiar with it and are usually not against acupuncture therapy. In this study, only one young female patient randomized to the acupuncture group refused acupuncture owing to a fear of needling. She was put in the control group then. Among the 80 SCI patients, 12 $(30 \%)$ cases in the control group and eight $(20 \%)$ in the acupuncture group discharged from hospital before their bladder balanced, and still needed intermittent catheterization. They were all with complete spinal cord injury (Frankel A), six were upper motor neuron lesions with severe detrusor-sphincter dyssynergia and 14 were lower motor neuron lesions with persistent atonic bladder. They were excluded in the final statistical analysis. Therefore, a total of 60 cases $(25$ Frankel A and 35 Frankel B) who had ultimately achieved balanced voiding were retained in this study, 28 cases in the control group and 32 cases in the acupuncture group.

In order to evaluate the difference of therapeutic effects between upper and lower motor neuron type neurogenic bladder, subjects in the control and acupuncture group were also divided into two subgroups, according to the level of spinal injury above or below T11. There were 34 patients with upper motor neuron lesions (13 Frankel A, 21 Frankel B) and 26 patients with lower motor neuron lesions (12 Frankel A, 14 Frankel B).
One of the impracticalities of the acupuncture method is that some people do not like needles. According to the traditional Chinese Medicine, Conception Vessel 3,4 $\left(\mathrm{CV}_{3}, \mathrm{CV}_{4}\right)$ and bilateral Urinary Bladder 31-34 (UB 31-34) are a benefit to the organ disorders in the pelvic cavity. We selected four acupoints only in this study (as shown in Figure 1) because too many more acupoints might increase patient's suffering. One patient was placed in the supine position, a needle was inserted according to the routine procedure into the acupoint of Guan Yuan $\left(\mathrm{CV}_{4}\right.$, midpoint from umbilicus to symphysis pubis at midline) and Zhongji $\left(\mathrm{CV}_{3}\right.$, upper one third from $\mathrm{CV}_{4}$ to symphysis pubis at midline). Then the patient was placed on a side-lying position. The needle was inserted into bilateral Tzu Liao (UB32) which was located just at the sacral foramen S2. While inserted, the needle should be manipulated till the feeling of 'De Qi', i.e. either a visible localized muscle contraction or needle sensation of soreness, heaviness or numbness at a local insertion site. Some of the patients even had the needling sensation extending into the penis or scrotum after needle insertion to the acupoint of $\mathrm{CV}_{3}$ or $\mathrm{CV}_{4}$, and some patients had the same needling sensation extending to the perineum after needle insertion to the acupoint of UB32.

The pulsator 3000 electroacupuncture apparatus was applied, attaching the electrode to the needle holder by alligator clip. The frequency of the pulse was $20-30 \mathrm{~Hz}$ with a pulse duration of $200 \mathrm{usec}$. The intensity of stimulus was around $30-50 \mathrm{mAmps}$, depending on the patient's tolerance. Each session lasted for $15 \mathrm{~min}$. Patients received electroacupuncture treatment 4-5 sessions each week, till their bladders were balanced. The bladder was considered to be balanced when (1) the patient could easily pass adequate urine at low pressure, (2) residual urine
A.

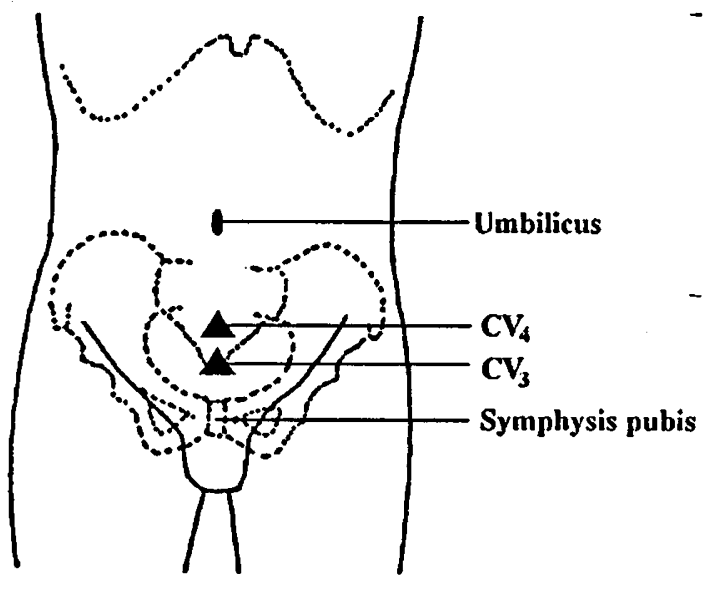

B.

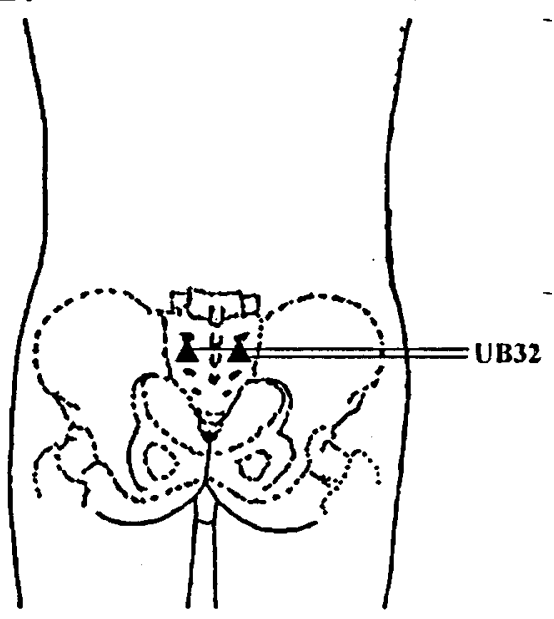

Figure 1 Location of acupoint $\mathrm{CV}_{3}, \mathrm{CV}_{4}$ and UB32. (A) Anterior view. CV4 is located on the midpoint from umbilicus to Symphysis pubis. $\mathrm{CV}_{3}$ is located on the upper one third from $\mathrm{CV}_{4}$ to Symphysis pubis. (B) Posterior view. UB $\mathrm{B}_{32}$ is located at the intervertebral foramen between $\mathrm{S} 2$ and $\mathrm{S} 3$ 
was approximately $100 \mathrm{ml}$ or less, and (3) there were no urinary tract infection.

In the acupuncture group, patients received acupuncture therapy as soon as their condition permitted. In order to evaluate the timing effect of electroacupuncture in the management of neurogenic bladder, 18 patients receiving electroacupuncture within the first 3 weeks of spinal cord injury were assigned as Group A, and the other 14 patients receiving electroacupuncture after 3 weeks of spinal cord injury were put in Group B. The reasons for delaying acupuncture in group B patients were (1) significant spinal fracture dislocation with traction application for about 2 weeks before surgical fixation (five patients); (2) aspiration pneumonia or urinary tract infection with fever (five patients); (3) referred from other hospitals 1 month after spinal injury (four patients).

Twenty cases of the acupuncture group agreed to receive urodynamic assessment, simultaneously monitoring the bladder, urethral and sphincter activity just before and after the first acupuncture therapy as well as after the bladder balanced.

Table 1 Clinical information of the control group and acupuncture group

\begin{tabular}{lcc}
\hline & $\begin{array}{c}\text { Control group } \\
(n=28)\end{array}$ & $\begin{array}{c}\text { Acupuncture group } \\
(n=32)\end{array}$ \\
\hline Age (mean \pm S.D.) & $34.3 \pm 11.6$ & $39.4 \pm 12.1$ \\
$\quad($ range $)$ & $18-68$ & $25-70$ \\
Sex (M:F) & $23: 5$ & $24: 8$ \\
Duration of SCI & $26.1 \pm 12.1$ & $23.7 \pm 12.8$ \\
$\quad$ (days) & & \\
Level of spine injury & & 18 \\
$\quad$ above T11 & 16 & 14 \\
below T11 & 12 & \\
\hline
\end{tabular}

Statistically, students $t$-test was used. $P<0.05$ was considered statistically significant.

\section{Results}

Among the 80 SCI patients with neurogenic bladder, 28 $(70 \%)$ cases in the control group and $32(80 \%)$ cases in the acupuncture group achieved ultimately balanced voiding and were selected for the final analysis. Clinical information of the acupuncture and control group were summarized in Table 1. Obviously spinal cord injury occurred predominately in the young male adults. There was no significant difference of mean age, sex ratio, duration of SCI and the level of spinal injury between the acupuncture and control group. Comparison of the total days needed to reach bladder balance showed that the acupuncture group appeared to acheive a significant shortening of the total days achieving bladder balance than the control group, either in upper motor neuron lesions or in lower motor neuron lesions, $57.1 \pm 22.5 \mathrm{vs}$ $85.2 \pm 27.4$ days $(P<0.005)$ and $55.4 \pm 22.6$ vs $83.4 \pm 26.1$ days $(P<0.01)$ respectively (Table 2$)$. However, no significant difference was found between upper and lower motor neuron lesions, both in the control group and in the acupuncture group (Table 2).

As shown in Table 3, Group A patients who received acupuncture therapy within 3 weeks after spinal injury revealed a significantly shorter period to reach a balanced bladder, as compared to Group B who received acupuncture therapy after 3 weeks of spinal injury, $46.6 \pm 13.2$ days vs $65.8 \pm 15.4$ days $(P<0.005)$.

Among the 20 cases who underwent urodynamic study to evaluate electroacupuncture effects, no detrusor contraction was found in the initial cystometrogram of all the lower motor neuron lesions and half of the upper motor neuron lesions. All of them were unable to void by themselves. Most of them showed no significant urodynamic change before and after the

Table 2 Comparison of the outcome of neurogenic bladder between control and acupuncture group in the patients with complete spinal cord injury

\begin{tabular}{|c|c|c|c|}
\hline & Control group & Acupuncture group & $p$ \\
\hline \multicolumn{4}{|l|}{ Case No. } \\
\hline Upper motor neuron lesions & 16 & 18 & \\
\hline Lower motor neuron lesions & 12 & 14 & \\
\hline \multicolumn{4}{|c|}{ Period from SCI to self voiding (days) } \\
\hline Upper motor neuron lesion & $42.4 \pm 15.1$ & $33.6 \pm 15.0$ & $<0.05$ \\
\hline Lower motor neuron lesion & $44.1 \pm 14.8$ & $31.5 \pm 14.2$ & $<0.05$ \\
\hline$P$ & $>\overline{0} .1$ & $>\overline{0} .1$ & \\
\hline \multicolumn{4}{|c|}{ Period from self-voiding to bladder balanced (days) } \\
\hline Upper motor neuron lesion & $43.4 \pm 16.8$ & $24.5 \pm 14.3$ & $<0.005$ \\
\hline Lower motor neuron lesion & $39.6 \pm 15.6$ & $24.2 \pm 11.8$ & $<0.01$ \\
\hline$P$ & $>0.1$ & $>0.1$ & \\
\hline \multicolumn{4}{|c|}{ Total days needed to reach bladder balanced } \\
\hline Upper motor neuron lesion & $85.2 \pm 27.4$ & $57.1 \pm 22.5$ & $<0.005$ \\
\hline Lower motor neuron lesion & $83.4 \pm 26.1$ & $55.4 \pm 22.6$ & $<0.01$ \\
\hline$P$ & $>\overline{0} .1$ & $>\overline{0} .1$ & \\
\hline
\end{tabular}


Table 3 The effect of timing of acupuncture in bladder training

\begin{tabular}{llc}
\hline & $\begin{array}{c}\text { Group A } \\
(n=18)\end{array}$ & $\begin{array}{c}\text { Group } \\
(n=18)\end{array}$ \\
\hline Period from SCI to self-voiding (days) & $25.2 \pm 10.4$ & $38.2 \pm 11.3$ \\
Period from self-voiding to bladder balanced (days) & $22.4 \pm 10.6$ & $<0.005$ \\
Total days needed to reach bladder balanced & $46.6 \pm 13.2$ & $28.9 \pm 10.8$ \\
\hline
\end{tabular}

*Group A: start acupuncture within the first 3 weeks of SCI. Group B: start acupuncture after 3 weeks of SCI

first acupuncture therapy, only four patients showed increasing detrusor contractions just after the first electroacupuncture therapy. However, at the time of balanced bladder achieved, the contractility of detrusor was shown to be improved with maximal detrusor pressure of $30 \pm 7.5 \mathrm{~cm} \mathrm{H}_{2} \mathrm{O}$ in upper motor neuron lesions and $23 \pm 7.2 \mathrm{~cm} \mathrm{H}_{2} \mathrm{O}$ in lower motor neuron lesions. The maximal cystometric bladder capacity was $502 \pm 102 \mathrm{ml}$ in the upper motor neuron lesions and $506 \pm 125 \mathrm{ml}$ in the lower motor neuron lesions. The maximal voiding pressure was $52 \pm 11.3$ $\mathrm{cmH}_{2} \mathrm{O}$ and $50 \pm 12.1 \mathrm{cmH}_{2} \mathrm{O}$ in the upper and lower motor neuron lesions respectively and their residual urine were all less than $100 \mathrm{cc}$.

\section{Discussion}

Spinal cord injury with neurogenic bladder was an important task for rehabilitation. Only about twothirds could ultimately achieve balanced voiding by conventional methods. In the control group of this study, the total days needed to reach bladder balanced for upper and lower motor neuron lesions were $85.2 \pm 27.4$ and $83.4 \pm 26.1$ days respectively. The results were the same as previous reports. ${ }^{1,2}$ No significant difference between upper and lower motor neuron lesion was found.

Acupuncture is an important branch of traditional Chinese Medicine. Research has confirmed its good analgesic effect through a noxious stimulus which induces endogenous product of pain relief substances. ${ }^{11}$ Experiments on animals have verified the positive effects on the circulation, digestive and urinary systems. ${ }^{12}$ Acupuncture therapy for bladder dysfunction is to achieve bladder emptying in cases or urinary retention, and to inhibit detrusor hyperactivity in cases of enuresis or incontinence. ${ }^{13-16}$ Both $\mathrm{Yang}^{13}$ and $\mathrm{Hou}^{14}$ reported a $100 \%$ success rate in the treatment of postpartum dysuria by using acupuncture. $\mathrm{An}^{15}$ and $\operatorname{Ran}^{16}$ used acupuncture therapy in the management of spinal lesion with dysuria, a $93 \%$ effective rate was obtained. In this study, the acupuncture group had significantly shortened the total days to reach a bladder balance as compared to the control group. These findings made acupuncture a positive role in the management of neurogenic bladder.

Although acupuncture has been used for treatment on a variety of diseases in China for over 2000 years, the true physiology of its role in the urological disorders is still obscure. $\mathrm{Cai}^{17}$ has reported the correlation of acupuncture and the nervous system. He pointed out that there are peripheral nerves and terminals at each acupuncture point. In addition, there are high concentrations of neurotransmitters and hormones within the acupuncture points of human beings. These hormones might cause inhibition, excitation or both to the target neurons. Pomeranz ${ }^{18}$ in his study mentioned that a weak DC electric field might enhance motor nerve regeneration and sensory nerve sprouting in adult rats. Acupuncture needle insertion could create currents of injury sufficient to supply a similar weak DC field. Consequently, electroacupuncture through acupuncture loci might activate afferent nerve fibers and initiate a number of physiological mechanisms.

Some people might consider that a complete spinal cord injury with areflexic bladder could not achieve bladder contractility. In fact, Wyndaele et $a l^{19}$ demonstrated the existence of an afferent nerve pathway from the bladder to the cerebral cortex in over one third of the clinically complete spinal cord injured patients. Furthermore, as described by Dimitrijevic, $^{20}$ a majority of so-called clinically complete injuries are actually subclinically incomplete, retaining significant communication between segments above and below the level of injury. Electroacupuncture could induce detrusor contractility through activating the existing nerve fibers, which was why our clinically complete SCI patients could achieve a balanced bladder by electroacupuncture. In contrast, those cases which did not achieve balanced bladder eventually in this study might be truly complete SCI patients. Therefore, it was supposed that electroacupuncture could enable the bladder to achieve balanced status in both upper and lower motor neuron lesions. The possible mechanism of acupuncture in the management of spinal cord injured patients with neurogenic bladder was implied as: (1) Activation of segmental or suprasegmental reflex pathways after stimulation of afferent nerve fibers; (2) Hormonal change, influenced through the ascending pathways to the hypothalamus; (3) Reflex activation of autonomic efferents to the bladder.

In this study, we selected four acupoints (as shown in Figure 1) for management of the neurogenic bladder in SCI patients. From the view of neuroanatomy, CV3 and CV4 are in the front of the body, with location just the above the bladder in an umbilical line. Needle 
insertion at these two acupoints might stimulate the afferent fiber, activate the autonomic component, and initiate controlled bladder activity to aid evacuation. The bilateral UB32 are located at the back around the sacral foramen S2, the sacral root S3 passes through here and then innervates to the bladder. Although it is known that S3 is the main root for detrusor contraction, acupuncture around the sacral foramen S2 obviously is also able to induce detrusor contractility. The improvement of detrusor contractility shown in urodynamic study at the time of bladder balance and the shorter time to achieve a balanced bladder further supported our hypothesis.

In the acupuncture therapy group, we also found those who received electroacupuncture therapy within 3 weeks of spinal cord injury had significantly shortened the total days to achieve a balanced bladder, as compared to those who received acupuncture therapy after 3 weeks of a spinal cord injury $(P<0.005)$. These findings implied that the earlier the patient received acupuncture therapy, the sooner the patient could void spontaneously without catheterization.

In summary, acupuncture therapy had its effect in the management of spinal cord injured patients with the neurogenic bladder. When to start acupuncture therapy also played an important role. The earlier the patients received acupuncture therapy, the sooner the patients achieved a bladder balance. On the other hand, complete SCI patients with severe detrusorsphincter dyssynergia or persistent areflexic bladder were not affected by acupuncture. However, we cannot draw any conclusions regarding efficacy, because we have no urodynamic data from the control group in this study. One must assume that similar changes have occurred in the control group, as patients of this group also achieved balanced voiding although somewhat later. Further investigation is being conducted to evaluate this result in depth.

\section{References}

1 Arnold EP, Fukui J, Anthony A, Utley WLF. Bladder function following spinal cord injury, a urodynamic analysis of the outcome. Br J Urol 1984; 56: $172-177$.
2 Cheng PT, Lee CC. Rehabilitation outcome of neurogenic bladder in lower spinal cord injured patients. Chang Gung Med J 1988; 11: $116-119$.

3 Madersbacher H. Intravesical electrical stimulation for the rehabilitation of the neurogenic bladder. Paraplegia 1990; 28: $349-352$.

4 Dees JE. Contraction of the urinary bladder produced by electric stimulation: preliminary report. Invest Urol 1965; 2: 539.

5 Fall M, Carlsson CA, Erlandson BE. Electrical stimulation in interstitial cystitis. J Urol 1980; 123: 192.

6 Tanagho EA, Schmidt RA. Electrical stimulation in the clinical management of the neurogenic bladder. J Urol 1988; 140: 1331 1339.

7 Tanagho EA, Schmidt RA, Orvis BR. Neural stimulation for control of voiding dysfunction: a preliminary report in 22 patients with serious neuropathic voiding disorders. J Urol 1989; 142: 340 - 345 .

8 Sauerwein D et al. Extradural implantation of sacral anterior root stimulators. J Neurol Neurosurg Psych 1990; 53: 681-684.

9 Carlsson CA, Fall M. Electrical stimulation of the conus medullaris for bladder emptying in a paraplegic. Paraplegia 1984; 22: 87 - 91 .

10 Ohlsson BL, Fall M, Frankenberg-Sommar S. Effects of external and direct pudendal nerve maximal electrical stimulation in the treatment of the uninhibited overactive bladder. Br J Urol 1989; 64: $374-380$.

11 Han JS, Sun SL. Differential release of enkephalin and dynorphin by low and high frequency electroacupuncture in the central nervous system. Acupuncture Sci Intern J 1990; 1: 19-24.

$12 \mathrm{Wu}$ DZ. Acupuncture and neurophysiology. Chin Neurol Neurosurg 1990; 92: 13-25.

13 Yang DL. Acupuncture therapy in 49 cases of postpartum urinary retention. J Trad Chin Med 1985; 5: 26.

14 Hou XJ. 30 cases of postpartum dysuria treated with acupuncture. J Trad Chin Med 1989; 9: 186.

$15 \mathrm{An} \mathrm{XC}$ et al. Acupuncture treatment for disturbances in urination and defecation from sacral cryptorachischisis - a clinical observation of 254 cases. J Trad Chin Med 1986; 6: $95-$ 98.

16 Ran C et al. Acupuncture treatment of spinal paraplegia with acupoints selected, basing on neuroanatomy. Word J Acup-Mox 1992 2: 3-9.

17 Cai W. Acupuncture and the nervous system. Am J Chinese Med 1992; XX: $331-337$.

18 Pomeranz B. Weak DC electric fields enhance motor nerve regeneration and sensory nerve sprouting in adult rats: a model for acupuncture effect. Acupuncture Electro-Therap Res Int $J$ 1988; 13: 208

19 Wyndaele JJ. Investigation of the afferent nerves of the lower urinary tract in patients with complete and incomplete spinal cord injury. Paraplegia 1991; 29: $490-494$.

20 Dimitrijevic MR. Residual motor function in spinal cord injury. In Waxman SG (ed): Functional recovery in neurological disease. Advances in Neurology 1988; 47: 139-155. 